\title{
THE RAMANUJAN-SERRE DIFFERENTIAL OPERATORS AND CERTAIN ELLIPTIC CURVES
}

\author{
MASANOBU KANEKO AND YUICHI SAKAI \\ (Communicated by Ken Ono)
}

\begin{abstract}
For several congruence subgroups of low levels and their conjugates, we derive differential equations satisfied by the Eisenstein series of weight 4 and relate them to elliptic curves whose associated newforms of weight 2 constitute the list of Martin and Ono of newforms given by etaproducts/quotients.
\end{abstract}

\section{INTRODUCTION}

Let

$$
E_{4}(\tau)=1+240 \sum_{n=1}^{\infty}\left(\sum_{d \mid n} d^{3}\right) q^{n} \quad\left(q=e^{2 \pi i \tau}, \tau \in \mathfrak{H}: \text { the upper-half plane }\right)
$$

and

$$
E_{6}(\tau)=1-504 \sum_{n=1}^{\infty}\left(\sum_{d \mid n} d^{5}\right) q^{n}
$$

be the standard Eisenstein series of weights 4 and 6 on the modular group $\mathrm{SL}_{2}(\mathbb{Z})$. The classical relation

$$
E_{4}(\tau)^{3}-E_{6}(\tau)^{2}=1728 \Delta(\tau),
$$

where $\Delta(\tau)=\eta(\tau)^{24}$ with $\eta(\tau)=q^{1 / 24} \prod_{n=1}^{\infty}\left(1-q^{n}\right)$ the Dedekind eta function, can be seen as the elliptic curve

$$
y^{2}=x^{3}-1728
$$

being parametrized by modular functions

$$
x=\frac{E_{4}(\tau)}{\eta(\tau)^{8}}=\sqrt[3]{j(\tau)}, y=\frac{E_{6}(\tau)}{\eta(\tau)^{12}}=\sqrt{j(\tau)-1728},
$$

where $j(\tau)=E_{4}(\tau)^{3} / \Delta(\tau)$ is the elliptic modular function.

In [1, Pavel Guerzhoy viewed this parametrization as a differential equation satisfied by $E_{4}(\tau)$ and its derivative $\partial\left(E_{4}(\tau)\right)=E_{6}(\tau)$, where $\partial$ is (a suitable multiple of) the Ramanujan-Serre differential operator, and investigated from this viewpoint certain Kummer type congruences satisfied by values of derivatives of modular forms. We note that the elliptic curve $(\mathbb{1})$ is isomorphic (over $\mathbb{Q}$ ) to the minimal curve

$$
y^{2}=x^{3}-27
$$

Received by the editors January 3, 2012 .

2010 Mathematics Subject Classification. Primary 11F11, 11F25; Secondary 11G05, 11F20. 
of conductor 36 and that the associated newform of weight 2 and level 36 is given by the eta product $\eta(6 \tau)^{4}$, which is seen (as will be justified in the sequel) to be derived from $\Delta(\tau)=\eta(\tau)^{24}$ as $\eta(6 \cdot \tau)^{24 / 6}$, where the number 6 is half the weight of $\Delta(\tau)$.

In the present paper, we show that there exist similar procedures when we consider the Ramanujan-Serre differential operator for several congruence subgroups of low levels and that the list in the paper [3] of Yves Martin and Ken Ono of weight 2 newforms given by eta-products (or quotients) can be recovered.

\section{MAIN RESUlT}

Let $N$ be a positive integer and

$$
\Gamma_{0}(N)=\left\{\left(\begin{array}{ll}
a & b \\
c & d
\end{array}\right) \in \mathrm{SL}_{2}(\mathbb{Z}) \mid c \equiv 0 \bmod N\right\}
$$

be the Hecke congruence subgroup of level $N$. We start with the eta-product $\Delta_{N}(\tau)$ defined by

$$
\Delta_{N}(\tau)=\left(\prod_{d \mid N} \eta(d \tau)\right)^{24 / \mu_{N}},
$$

where $\mu_{N}$ is the index of $\Gamma_{0}(N)$ in $\mathrm{SL}_{2}(\mathbb{Z})$, which is given by

$$
\mu_{N}=\left[\mathrm{SL}_{2}(\mathbb{Z}): \Gamma_{0}(N)\right]=N \prod_{\substack{p \mid N \\ p: \text { prime }}}\left(1+\frac{1}{p}\right)
$$

The weight $k_{N}$ of $\Delta_{N}(\tau)$ is

$$
k_{N}=\frac{12 \sigma_{0}(N)}{\mu_{N}}, \quad \sigma_{0}(N)=\text { the number of divisors of } N,
$$

and the $q$-expansion of $\Delta_{N}(\tau)$ begins at $q^{h_{N}}$, where

$$
h_{N}=\frac{\sigma_{1}(N)}{\mu_{N}}, \quad \sigma_{1}(N)=\sum_{d \mid N} d .
$$

Using these explicit formulae it is easy to compute all values of $N$ for which the weight $k_{N}$ is an even integer and the exponent $h_{N}$ is an integer. Here is the list (we also give values of $\mu_{N}$ for convenience):

\begin{tabular}{c|c|c|c|c|c|c|c|c}
$N$ & 1 & 2 & 3 & 5 & 6 & 11 & 14 & 15 \\
\hline \hline$k_{N}$ & 12 & 8 & 6 & 4 & 4 & 2 & 2 & 2 \\
\hline$h_{N}$ & 1 & 1 & 1 & 1 & 1 & 1 & 1 & 1 \\
\hline$\mu_{N}$ & 1 & 3 & 4 & 6 & 12 & 12 & 24 & 24
\end{tabular}

In all cases the exponent $24 / \mu_{N}$ in the definition of $\Delta_{N}(\tau)$ is a positive integer. For these $N$, the form $\Delta_{N}(\tau)$ is a cusp form of weight $k_{N}$ on the group $\Gamma_{0}(N)$. To see this we can use, for instance, the theorem of Honda-Miyawaki [2, Theorem 1], but this is standard anyway and we omit the details here.

Let $P_{N}(\tau)$ be the logarithmic derivative of $\Delta_{N}(\tau)$,

$$
P_{N}(\tau)=q \frac{d}{d q} \log \Delta_{N}(\tau)=\frac{1}{\mu_{N}} \sum_{d \mid N} d E_{2}(d \tau),
$$


where

$$
E_{2}(\tau)=1-24 \sum_{n=1}^{\infty}\left(\sum_{d \mid n} d\right) q^{n}
$$

is the ("quasimodular") Eisenstein series of weight 2 on $\mathrm{SL}_{2}(\mathbb{Z})$. We introduce the (Ramanujan-Serre) differential operator $\partial_{k}^{(N)}$ defined by

$$
\partial_{k}^{(N)}(f)(\tau)=\frac{k_{N}}{4} \cdot q \frac{d f}{d q}(\tau)-\frac{k}{4} \cdot P_{N}(\tau) f(\tau) \quad\left(q \frac{d}{d q}=\frac{1}{2 \pi \sqrt{-1}} \frac{d}{d \tau}\right)
$$

for modular forms $f(\tau)$ of weight $k$. Note that we choose a different normalization from the standard one by the factor $k_{N} / 4$ (in the classical case of $\mathrm{SL}_{2}(\mathbb{Z})$ the most commonly used is $\left.\partial_{k}(f)(\tau)=q d f / d q-k E_{2} f / 12\right)$. From the transformation property of $P_{N}(\tau)$ (which is quasimodular of weight 2), we see that if $f$ is modular of weight $k$ on $\Gamma_{0}(N)$, then $\partial_{k}^{(N)}(f)$ is modular of weight $k+2$ on $\Gamma_{0}(N)$. (A quick way to see this is to consider the derivative of the weight 0 modular function $f(\tau)^{k_{N}} / \Delta_{N}(\tau)^{k}$, which is modular of weight 2.)

We further consider the group $\Gamma_{0}^{\sharp}(N)$ which is conjugate to $\Gamma_{0}(N)$ by $\left(\begin{array}{ll}2 & 1 \\ 0 & 2\end{array}\right)$ :

$$
\Gamma_{0}^{\sharp}(N)=\left(\begin{array}{ll}
2 & 1 \\
0 & 2
\end{array}\right)^{-1} \Gamma_{0}(N)\left(\begin{array}{ll}
2 & 1 \\
0 & 2
\end{array}\right) .
$$

This group contains $\Gamma_{0}(4 N)$ as a subgroup. Let

$$
\Delta_{N}^{\sharp}(\tau)=-\Delta_{N}(\tau+1 / 2)
$$

and

$$
P_{N}^{\sharp}(\tau)=q \frac{d}{d q} \log \Delta_{N}^{\sharp}(\tau) .
$$

Also let $\partial_{k}^{(N \sharp)}$ be defined by

$$
\partial_{k}^{(N \sharp)}(f)=\frac{k_{N}}{4} \cdot q \frac{d f}{d q}-\frac{k}{4} \cdot P_{N}^{\sharp} \cdot f
$$

for a form $f$ of weight $k$. Here and in the following, we sometimes suppress the variable $\tau$ of modular forms. Our main result is the following theorem.

Theorem. (i) For $N=1,2,3,5,6$, let $Q_{N}=Q_{N}(\tau)$ be any one of the Eisenstein series of weight 4 on $\Gamma_{0}(N)$ associated to cusps. Then the forms $Q_{N}$ and $\partial_{4}^{(N)}\left(Q_{N}\right)$ satisfy a homogeneous (with respect to weights) polynomial relation of degree 3 (and of weight 12 ) over $\mathbb{Q}\left[\Delta_{N}\right]$. This relation can be written in the form that the pair

$$
\left(\frac{Q_{N}}{\Delta_{N}^{4 / k_{N}}}, \frac{\partial_{4}^{(N)}\left(Q_{N}\right)}{\Delta_{N}^{6 / k_{N}}}\right)
$$

of modular functions satisfies the equation of an elliptic curve $E_{N}$ over $\mathbb{Q}$. The isomorphism class of $E_{N}$ over $\mathbb{Q}$ is independent of the choice of $Q_{N}$. The conductor of the minimal model of $E_{N}$ is $k_{N}^{2} N / 4$, and the associated newform of weight 2 is given by

$$
\Delta_{N}\left(\frac{k_{N}}{2} \tau\right)^{2 / k_{N}}
$$


(ii) Suppose $N=1,2,5,6$, and let $Q_{N}$ be as in (i). Put $Q_{N}^{\sharp}=Q_{N}(\tau+1 / 2)$. Then we obtain a similar differential equation for $Q_{N}^{\sharp}$ and an elliptic curve $E_{N}^{\sharp}$ whose equation is satisfied by

$$
\left(\frac{Q_{N}^{\sharp}}{\left(\Delta_{N}^{\sharp}\right)^{4 / k_{N}}}, \frac{\partial_{4}^{(N \sharp)}\left(Q_{N}^{\sharp}\right)}{\left(\Delta_{N}^{\sharp}\right)^{6 / k_{N}}}\right) .
$$

The conductor of the minimal model of $E_{N}^{\sharp}$ is $k_{N}^{2} N$ when $N$ is odd and $k_{N}^{2} N / 2$ when $N$ is even, and the associated newform of weight 2 is given by

$$
\Delta_{N}^{\sharp}\left(\frac{k_{N}}{2} \tau\right)^{2 / k_{N}} .
$$

Remark. All powers of $\Delta_{N}$ and $\Delta_{N}^{\sharp}$ appearing in the theorem become integral powers of products of etas.

Before giving the proof, we tabulate in two tables the differential equations, the associated elliptic curves (minimal models) and the newforms. The coefficients of the differential equations depend on normalizations of the Eisenstein series of weight 4. We find that the isomorphism classes of resulting elliptic curves are independent, not only of the choices of normalizing constants but also of the choices of the Eisenstein series at cusps, and rather surprisingly, we can choose a normalization of each Eisenstein series to obtain the same equation. For $N=1$, there is only one cusp at $i \infty$, and for $N=2,3,5$, there are two inequivalent cusps at $i \infty$ and 0 . For $N=6$, there are four cusps, $1 / 2$ and $1 / 3$ being the cusps other than $i \infty$ and 0 . We take the Eisenstein series of weight 4 associated to cusps $i \infty$ and 0 as

$$
E_{4, N}^{i \infty}(\tau)=c_{N} \sum_{d \mid N} \frac{\mu(d)}{d^{4}} E_{4}\left(\frac{N}{d} \tau\right)
$$

and

$$
E_{4, N}^{0}(\tau)=\frac{c_{N}}{N^{2}} \sum_{d \mid N} \mu(d) E_{4}(d \tau)
$$

respectively, where $c_{N}$ is the normalizing constant

$$
c_{N}=\prod_{\substack{p \mid N \\ p: \text { prime }}}\left(1-\frac{1}{p^{4}}\right)^{-1}
$$

and $\mu(d)$ is the Möbius function. When $N=6$, as two more Eisenstein series $E_{4,6}^{1 / 2}(\tau)$ and $E_{4,6}^{1 / 3}(\tau)$ associated to cusps $1 / 2$ and $1 / 3$ respectively, we take

$E_{4,6}^{1 / 2}(\tau)=\frac{3^{2}}{3^{4}-1}\left(E_{4,2}^{i \infty}(\tau)-E_{4,2}^{i \infty}(3 \tau)\right)$ and $E_{4,6}^{1 / 3}(\tau)=\frac{2^{2}}{2^{4}-1}\left(E_{4,3}^{i \infty}(\tau)-E_{4,3}^{i \infty}(2 \tau)\right)$.

Table 1 below is the list of initial eta-products and the differential equations satisfied by Eisenstein series, and Table 2 gives minimal models of associated elliptic curves, their conductors, and newforms of weight 2 .

Proof of the Theorem. In each case in (i), once a candidate of the differential equation is found, it becomes a matter of computing enough Fourier series by computer in order to rigorously prove that the equation holds true. This is because each term of the differential equations in Table 1 is a holomorphic modular form of weight 12 on each congruence subgroup, and hence, as is well known (the valence formula or the Riemann-Roch theorem), the vanishing of Fourier coefficients up to $q^{\mu_{N}}$ ensures 
TABLE 1

\begin{tabular}{c|c|c} 
Group & $\Delta_{N}$ or $\Delta_{N}^{\sharp}$ & Differential equation \\
\hline \hline$\Gamma_{0}(1)$ & $\eta(\tau)^{24}$ & $\partial_{4}^{(1)}\left(Q_{1}\right)^{2}=Q_{1}^{3}-1728 \Delta_{1}$ \\
\hline$\Gamma_{0}(2)$ & $\eta(\tau)^{8} \eta(2 \tau)^{8}$ & $\partial_{4}^{(2)}\left(Q_{2}\right)^{2}=Q_{2}^{3}+64 \Delta_{2} Q_{2}$ \\
\hline$\Gamma_{0}(3)$ & $\eta(\tau)^{6} \eta(3 \tau)^{6}$ & $\partial_{4}^{(3)}\left(Q_{3}\right)^{2}=Q_{3}^{3}+\frac{729}{4} \Delta_{3}^{2}$ \\
\hline$\Gamma_{0}(5)$ & $\eta(\tau)^{4} \eta(5 \tau)^{4}$ & $\partial_{4}^{(5)}\left(Q_{5}\right)^{2}=Q_{5}^{3}-\frac{89}{13} \Delta_{5} Q_{5}^{2}-\frac{3500}{169} \Delta_{5}^{2} Q_{5}-\frac{125000}{2197} \Delta_{5}^{3}$ \\
\hline$\Gamma_{0}(6)$ & $\eta(\tau)^{2} \eta(2 \tau)^{2} \eta(3 \tau)^{2} \eta(6 \tau)^{2}$ & $\partial_{4}^{(6)}\left(Q_{6}\right)^{2}=Q_{6}^{3}-\frac{23}{5} \Delta_{6} Q_{6}^{2}-\frac{432}{25} \Delta_{6}^{2} Q_{6}-\frac{1296}{125} \Delta_{6}^{3}$ \\
\hline$\Gamma_{0}^{\sharp}(1)$ & $\frac{\eta(2 \tau)^{72}}{\eta(\tau)^{24} \eta(4 \tau)^{24}}$ & $\partial_{4}^{(1 \sharp)}\left(Q_{1}^{\sharp}\right)^{2}=\left(Q_{1}^{\sharp}\right)^{3}+1728 \Delta_{1}^{\sharp}$ \\
\hline$\Gamma_{0}^{\sharp}(2)$ & $\frac{\eta(2 \tau)^{32}}{\eta(\tau)^{8} \eta(4 \tau)^{8}}$ & $\partial_{4}^{(2 \sharp)}\left(Q_{2}^{\sharp}\right)^{2}=\left(Q_{2}^{\sharp}\right)^{3}-64 \Delta_{2}^{\sharp} Q_{2}^{\sharp}$ \\
\hline$\Gamma_{0}^{\sharp}(5)$ & $\frac{\eta(2 \tau)^{2} \eta(10 \tau)^{12}}{\eta(\tau)^{4} \eta(4 \tau)^{4} \eta(5 \tau)^{4} \eta(20 \tau)^{4}}$ & $\partial_{4}^{(5 \sharp)}\left(Q_{5}^{\sharp}\right)^{2}=\left(Q_{5}^{\sharp}\right)^{3}+\frac{89}{13} \Delta_{5}^{\sharp}\left(Q_{5}^{\sharp}\right)^{2}-\frac{3500}{169}\left(\Delta_{5}^{\sharp}\right)^{2} Q_{5}^{\sharp}+\frac{125000}{2197}\left(\Delta_{5}^{\sharp}\right)^{3}$ \\
\hline$\Gamma_{0}^{\sharp}(6)$ & $\frac{\eta(2 \tau)^{8} \eta(6 \tau)^{8}}{\eta(\tau)^{2} \eta(3 \tau)^{2} \eta(4 \tau)^{2} \eta(12 \tau)^{2}}$ & $\partial_{4}^{(6 \sharp)}\left(Q_{6}^{\sharp}\right)^{2}=\left(Q_{6}^{\sharp}\right)^{3}+\frac{23}{5} \Delta_{6}^{\sharp}\left(Q_{6}^{\sharp}\right)^{2}-\frac{432}{25}\left(\Delta_{6}^{\sharp}\right)^{2} Q_{6}^{\sharp}+\frac{1296}{125}\left(\Delta_{6}^{\sharp}\right)^{3}$ \\
\hline
\end{tabular}

TABLE 2

\begin{tabular}{c|c|c|c} 
Group & Minimal model of $E_{N}$ or $E_{N}^{\sharp}$ & Conductor & Weight 2 new form \\
\hline \hline$\Gamma_{0}(1)$ & $y^{2}=x^{3}-27$ & 36 & $\eta(6 \tau)^{4}$ \\
\hline$\Gamma_{0}(2)$ & $y^{2}=x^{3}+4 x$ & 32 & $\eta(4 \tau)^{2} \eta(8 \tau)^{2}$ \\
\hline$\Gamma_{0}(3)$ & $y^{2}+y=x^{3}$ & 27 & $\eta(3 \tau)^{2} \eta(9 \tau)^{2}$ \\
\hline$\Gamma_{0}(5)$ & $y^{2}=x^{3}+x^{2}-36 x-140$ & 20 & $\eta(2 \tau)^{2} \eta(10 \tau)^{2}$ \\
\hline$\Gamma_{0}(6)$ & $y^{2}=x^{3}-x-24 x-36$ & 24 & $\eta(2 \tau) \eta(4 \tau) \eta(6 \tau) \eta(12 \tau)$ \\
\hline$\Gamma_{0}^{\sharp}(1)$ & $y^{2}=x^{3}+27$ & 144 & $\frac{\eta(12 \tau)^{12}}{\eta(6 \tau)^{4} \eta(24 \tau)^{4}}$ \\
\hline$\Gamma_{0}^{\sharp}(2)$ & $y^{2}=x^{3}-4 x$ & 64 & $\frac{\eta(8 \tau)^{8}}{\eta(4 \tau)^{2} \eta(16 \tau)^{2}}$ \\
\hline$\Gamma_{0}^{\sharp}(5)$ & $y^{2}=x^{3}-x^{2}-36 x+140$ & 80 & $\frac{\eta(4 \tau)^{6} \eta(20 \tau)^{6}}{\eta(2 \tau)^{2} \eta(8 \tau)^{2} \eta(10 \tau)^{2} \eta(40 \tau)^{2}}$ \\
\hline$\Gamma_{0}^{\sharp}(6)$ & $y^{2}=x^{3}+x-24 x+36$ & 48 & $\frac{\eta(4 \tau)^{4} \eta(12 \tau)^{4}}{\eta(2 \tau) \eta(6 \tau) \eta(8 \tau) \eta(24 \tau)}$ \\
\hline
\end{tabular}

that the function is identically zero. Computations of minimal models of elliptic curves and associated newforms are now standard, and several tables are available online. 
As for (ii), the differential equation for each $\Gamma_{0}^{\sharp}(N)$ is only a translation from that for $\Gamma_{0}(N)$ by $\tau \mapsto \tau+1 / 2$. We should note that the sign of the definition of $\Delta_{N}^{\sharp}(\tau)$ is essential. The computations of datas of elliptic curves are again standard. This completes the proof of the Theorem.

Remarks. 1) For the remaining cases $N=11,14,15$, the weight of $\Delta_{N}(\tau)$ is 2 , and this form itself gives the newform associated to an elliptic curve of conductor $N$. Together with these, the newforms we obtained in the Theorem coincide with the forms in the list of Martin and Ono in [3]. We are very curious to know why this happens and what is the reason behind this coincidence.

For $N=11,14,15$, the same procedure as used in the Theorem does not work for the Eisenstein series of weight 4 . However, we find that certain forms of weight 4 satisfy desired forms of differential equations. We briefly describe the form of weight 4 and the differential equation in each case. The forms $\Delta_{N}$ and $P_{N}$ and the operator $\partial_{k}^{(N)}$ are as before. We put for any integer $m$

$$
E_{2, m}(\tau)=\frac{m E_{2}(m \tau)-E_{2}(\tau)}{m-1} .
$$

For $N=11$, we let

$$
Q_{11}=E_{4,11}^{i \infty}(\tau)-\frac{121}{61} E_{2,11}(\tau) \Delta_{11}(\tau)-\frac{1274}{915} \Delta_{11}(\tau)^{2} .
$$

Then the equation

$$
\partial_{4}^{(11)}\left(Q_{11}\right)^{2}=Q_{11}^{3}-\frac{31}{3} \Delta_{11}^{4} Q_{11}-\frac{2501}{108} \Delta_{11}^{6}
$$

holds. The minimal model of the elliptic curve $y^{2}=x^{3}-\frac{31}{3} x-\frac{2501}{108}$ is

$$
y^{2}+y=x^{3}-x^{2}-10 x-20,
$$

which is of conductor 11 and the associated newform of weight 2 is $\Delta_{11}(\tau)=$ $\eta(\tau)^{2} \eta(11 \tau)^{2}$.

For $N=14$, let

$$
E_{4,14}^{i \infty}(\tau)=\frac{14^{4} E_{4}(14 \tau)-7^{4} E_{4}(7 \tau)-2^{4} E_{4}(2 \tau)+E_{4}(\tau)}{\left(7^{4}-1\right)\left(2^{4}-1\right)}
$$

and set

$$
Q_{14}=E_{4,14}^{i \infty}(\tau)-\frac{1}{900}\left(1001 E_{2,14}(\tau)-168 E_{2,7}(\tau)+73 E_{2,2}(\tau)\right) \Delta_{14}(\tau) .
$$

Then we have the equation

$$
\partial_{4}^{(14)}\left(Q_{14}\right)^{2}=Q_{14}^{3}-\frac{187}{100} \Delta_{14}^{2} Q_{14}^{2}+\frac{3528}{625} \Delta_{14}^{4} Q_{14}-\frac{3863552}{421875} \Delta_{14}^{6} .
$$

The minimal model of the elliptic curve $y^{2}=x^{3}-\frac{187}{100} x^{2}+\frac{3528}{625} x-\frac{3863552}{421875}$ is the conductor 14 curve

$$
y^{2}+x y+y=x^{3}+4 x-6,
$$

whose associated newform is $\Delta_{14}(\tau)=\eta(\tau) \eta(2 \tau) \eta(7 \tau) \eta(14 \tau)$. 
Finally, for $N=15$, let

$$
E_{4,15}^{i \infty}(\tau)=\frac{15^{4} E_{4}(15 \tau)-5^{4} E_{4}(5 \tau)-3^{4} E_{4}(3 \tau)+E_{4}(\tau)}{\left(5^{4}-1\right)\left(3^{4}-1\right)}
$$

and set

$$
Q_{15}=E_{4,15}^{i \infty}(\tau)-\frac{1}{208}\left(210 E_{2,15}(\tau)-10 E_{2,5}(\tau)+9 E_{2,3}(\tau)\right) \Delta_{15}(\tau)
$$

Then we have the equation

$$
\partial_{4}^{(15)}\left(Q_{15}\right)^{2}=Q_{15}^{3}-\frac{209}{104} \Delta_{15}^{2} Q_{15}^{2}-\frac{93825}{10816} \Delta_{15}^{4} Q_{15}+\frac{860625}{1124864} \Delta_{15}^{6} .
$$

The minimal model of the elliptic curve $y^{2}=x^{3}-\frac{209}{104} x^{2}-\frac{93825}{10816} x+\frac{860625}{1124864}$ is the conductor 15 curve

$$
y^{2}+x y+y=x^{3}+x^{2}-10 x-10,
$$

whose associated newform is $\Delta_{15}(\tau)=\eta(\tau) \eta(3 \tau) \eta(5 \tau) \eta(15 \tau)$.

2) In (ii) of the Theorem, the case $\Gamma_{0}^{\sharp}(3)$ is missing because the resulting differential equation is the same as that for $\Gamma_{0}(3)$. We have the relation

$$
\Delta_{3}^{\sharp}(3 \tau)^{1 / 3}=\Delta_{3}(3 \tau)^{1 / 3}+4 \Delta_{3}(12 \tau)^{1 / 3}
$$

between $\Delta_{3}^{\sharp}(3 \tau)^{1 / 3}$ and $\Delta_{3}(3 \tau)^{1 / 3}$.

3) For $N=1,5,6$, the elliptic curve obtained for $\Gamma_{0}^{\sharp}(N)$ is a quadratic twist of that for $\Gamma_{0}(N)$, whereas in the case $N=2$, we have a quartic twist.

\section{FurTher COMMENTS AND REMARKS}

1. We have no conceptual reason why we should have started with our etaproduct $\Delta_{N}(\tau)$. In fact, it is possible that there are more instances of obtaining elliptic curves in the way described in our theorem. For example, consider the case of $\Gamma_{0}(7)$. Let

$$
\Delta_{7}(\tau)=E_{1,(\overline{7})}(\tau) \eta(\tau)^{3} \eta(7 \tau)^{3}
$$

be the unique cusp form of weight 4 on $\Gamma_{0}(7)$ where

$$
E_{1,(\overline{7})}(\tau)=1+2 \sum_{n=1}^{\infty} \sum_{d \mid n}\left(\frac{d}{7}\right) q^{n}
$$

is the Eisenstein series of weight 1 with Legendre character $(\overline{7})$, and define $P_{7}$ and $\partial_{k}^{(7)}$ as before. Note that $\Delta_{7}(\tau)$ has a zero in the upper half-plane, and so the operator $\partial_{k}^{(7)}$ does not necessarily send holomorphic modular forms on $\Gamma_{0}(7)$ to holomorphic ones. But still, we have the differential equation

$$
\partial_{4}^{(7)}\left(Q_{7}\right)^{2}=Q_{7}^{3}-\frac{17}{10} \Delta_{7} Q_{7}^{2}-\frac{637}{100} \Delta_{7}^{2} Q_{7}-\frac{45619}{1000} \Delta_{7}^{3}
$$

for $Q_{7}=E_{4,7}^{i \infty}(\tau)$ or $E_{4,7}^{0}(\tau)$. The corresponding elliptic curve has the minimal model

$$
y^{2}=x^{3}+x^{2}-7 x-52
$$

of conductor $336=2^{4} \cdot 3 \cdot 7$. 
What is the class of elliptic curves over $\mathbb{Q}$ obtained in the way described in the present paper?

2. We searched for the power series solution

$$
a_{0}+a_{1} q+a_{2} q^{2}+a_{3} q^{3}+\cdots
$$

of each differential equation for $\Gamma_{0}(N)$ in the Theorem (the case $\Gamma_{0}^{\sharp}(N)$ being obtained from this by $q \rightarrow-q$ ). When $N=1,2,6$, no solution other than the Eisenstein series exists.

For $N=3$, we find one more solution,

$$
-27 \frac{\Delta_{3}(\tau)}{E_{2,3}^{i \infty}(\tau)}=-27 q+486 q^{2}-5103 q^{3}+43956 q^{4}-347490 q^{5}+\cdots,
$$

where

$$
E_{2,3}^{i \infty}(\tau)=\frac{1}{2}\left(3 E_{2}(3 \tau)-E_{2}(\tau)\right)
$$

is the Eisenstein series of weight 2. This solution is also of weight 4 but has a pole at an elliptic point.

When $N=5$, we find two more solutions other than the Eisenstein series:

$$
\begin{aligned}
& \left(-\frac{18}{13} \pm 2 i\right) \frac{j_{5}(\tau)-2 \pm 11 i}{j_{5}(\tau)+11 \mp 2 i} \cdot \Delta_{5}(\tau) \\
= & \left(-\frac{18}{13} \pm 2 i\right) q-\left(\frac{32}{13} \pm 52 i\right) q^{2}+\left(\frac{2044}{13} \pm 384 i\right) q^{3}-\left(\frac{19696}{13} \pm 1256 i\right) q^{4}+\cdots,
\end{aligned}
$$

where $i=\sqrt{-1}$ and

$$
j_{5}(\tau)=\eta(\tau)^{6} / \eta(5 \tau)^{6}=\frac{1}{q}-6+9 q+10 q^{2}-30 q^{3}+6 q^{4}-25 q^{5}+\cdots
$$

is the "Hauptmodul" for $\Gamma_{0}(5)$, which is also given in terms of the forms we have used as

$$
j_{5}(\tau)=\frac{E_{4,5}^{i \infty}(\tau)}{\Delta_{5}(\tau)}-\frac{125}{13} .
$$

These solutions are of weight 4 and have poles at elliptic points $\pm 2 / 5+i / 5$ where the function $j_{5}(\tau)$ assumes the values

$$
j_{5}\left( \pm \frac{2}{5}+\frac{i}{5}\right)=-11 \mp 2 i .
$$

The series obtained by dividing by the leading coefficient

$$
\frac{j_{5}(\tau)-2 \pm 11 i}{j_{5}(\tau)+11 \mp 2 i} \cdot \Delta_{5}(\tau)=q-(17 \mp 13 i) q^{2}+(93 \mp 143 i) q^{3}-(70 \mp 806 i) q^{4}+\cdots
$$

has its coefficients in the integer ring $\mathbb{Z}[i]$.

3. One should be able to pursue the congruences studied in the paper [1] of Guerzhoy (the case $N=1$ ), at least for $N=2,3$ where associated curves also have complex multiplication. We leave this task for interested readers.

\section{ACKNOWLEDGEMENTS}

It is a pleasure for the first-named author to thank Pavel Guerzhoy for explaining the viewpoint developed in [1]. Both authors thank Professor Guerzhoy for his interest and encouragemant regarding their work. 


\section{REFERENCES}

1. P. Guerzhoy, The Ramanujan differential operator, a certain CM elliptic curve and Kummer congruences, Compositio Mathematica, 141 (2005), no. 3, 583-590. MR2135278 (2005m:11083)

2. T. Honda and I. Miyawaki, Zeta-functions of elliptic curves of 2-power conductor, J. Math. Soc. Japan, 26 (1974), no. 2, 362-373. MR0360594 (50:13042)

3. Y. Martin and K. Ono, Eta-quotients and elliptic curves, Proc. Amer. Math. Soc., 125 (1997), 3169-3176. MR:1401749 (97m:11057)

Faculty of Mathematics, Kyushu University, Motooka 744, Nishi-ku, Fukuoka 8190395, JAPAN

E-mail address: mkaneko@math.kyushu-u.ac.jp

International Institute for Carbon-Neutral Energy Research, Kyushu University, Motooka 744, Nishi-Ku, FukUOKA 819-0395, JAPAN

Current address: Yokomizo 3012-2, Ooki-machi, Mizunuma-gun, Fukuoka 830-0405, Japan

E-mail address: dynamixaxs@gmail.com 